\title{
Simplified Design of Low-Delay Oversampled NPR GDFT Filterbanks
}

\author{
Bogdan Dumitrescu, ${ }^{1,2}$ Robert Bregović, ${ }^{1}$ and Tapio Saramäki ${ }^{1}$ \\ ${ }^{1}$ Institute of Signal Processing, Tampere University of Technology, P.O. Box 553, 33101 Tampere, Finland \\ ${ }^{2}$ Department of Automatic Control and Computers, "Politehnica" University of Bucharest, 060032 Bucharest, Romania
}

Received 01 September 2004; Revised 17 April 2005; Accepted 18 April 2005

We propose an efficient algorithm for designing the prototype filters of oversampled, near-perfect reconstruction (NPR), GDFT modulated filterbanks (FB) with arbitrary delay. We describe simplified conditions for imposing NPR, posed on the frequency response of the distortion transfer function and on the stopband attenuation of the prototype filters. Given the analysis prototype, we show that the minimization of the stopband energy of the synthesis prototype, subject to the simplified NPR constraints, can be expressed as a convex optimization problem. Our algorithm consists of initialization with the prototype of a near-orthogonal FBwhich can also be designed via convex optimization-and then successive optimization of the synthesis and analysis prototypes. We give design examples, discuss the properties of the obtained FBs, and present synthetic echo control experiments. The presented results show that, for a given delay, our algorithm produces FBs with significantly better properties than the near-orthogonal FBs.

Copyright ( 2006 Hindawi Publishing Corporation. All rights reserved.

\section{INTRODUCTION}

Recent studies [1-4] on subband adaptive filtering have shown that good performances and flexibility are obtained by using oversampled nearly prefect reconstruction filterbanks. Moreover, a low implementation complexity is ensured by uniform filterbanks whose filters are obtained by (complex) modulation from a single prototype; the filters have complex coefficients, but the prototype is real. In this paper, we give an efficient design algorithm for such filterbanks.

A general filterbank (FB) structure is presented in Figure 1. The FB is oversampled when the down-sampling factor $R$ is smaller than the number of channels $M$. The subband signals $x_{k}[n], k=0: M-1$, are processed by adaptive filtering or other type of algorithms; however, since here we are interested in a general design method, we ignore the subband processing and assume that $y_{k}[n]=x_{k}[n]$. We assume that all analysis filters $H_{k}(z)$ and synthesis filters $F_{k}(z), k=0: M-1$, have complex coefficients. Ideally, the passband of a filter has a width of $2 \pi / M$; more precisely, the passband of $H_{k}(z)$ or $F_{k}(z)$ covers the interval $[2 k \pi / M, 2(k+1) \pi / M]$, as shown in Figure 2(b).

If the input signal is real, then we can take $M$ even, and only the first $M / 2$ channels of the $\mathrm{FB}$ are necessary. In this case, the FB processes the frequencies between 0 and $\pi$; those from $-\pi$ to 0 are discarded; no information is lost, but the subband signals are complex. For such an FB, the output $y[n]$ is twice the real part of the sum of synthesis bank outputs.
(Real subband signals could be used, but with a downsampling factor $R$ twice smaller, as shown in [5].)

If the output signal is a delayed version of the input one, that is, $y[n]=x[n-D]$, where $D$ is a positive integer, then the FB is a perfect reconstruction (PR); theory of oversampled modulated PR FBs is discussed in [5-7]. Since subband processing changes the subband signals, near PR (NPR) FBs are more interesting for practical purposes; in NPR FBs, $y[n]$ approximates $x[n-D]$ in a sense that will be detailed later.

We consider generalized DFT (GDFT) modulated FBs, whose filters have the impulse responses

$$
\begin{aligned}
& h_{k}[n]=h[n] \mathrm{e}^{j \pi(2 k+1)(n-D / 2) / M}, \\
& f_{k}[n]=f[n] \mathrm{e}^{j \pi(2 k+1)(n-D / 2) / M},
\end{aligned}
$$

where $h[n]$ and $f[n]$ are the impulse responses of the analysis and, respectively, the synthesis prototypes. The prototypes are FIR filters of orders $N_{h}$ and $N_{f}$, with real coefficients; their transfer functions are denoted by $H(z)$ and $F(z)$, respectively. The idealized magnitude responses of the analysis prototype and filters are given in Figure 2. For the synthesis bank, the responses are similar.

A frequent choice is to use a single prototype for both the analysis and synthesis banks, such that $N_{h}=N_{f}$ and $f_{k}[n]=$ $h_{k}^{*}\left[N_{h}-n\right]$. A near-orthogonal FB is obtained (or orthogonal, 


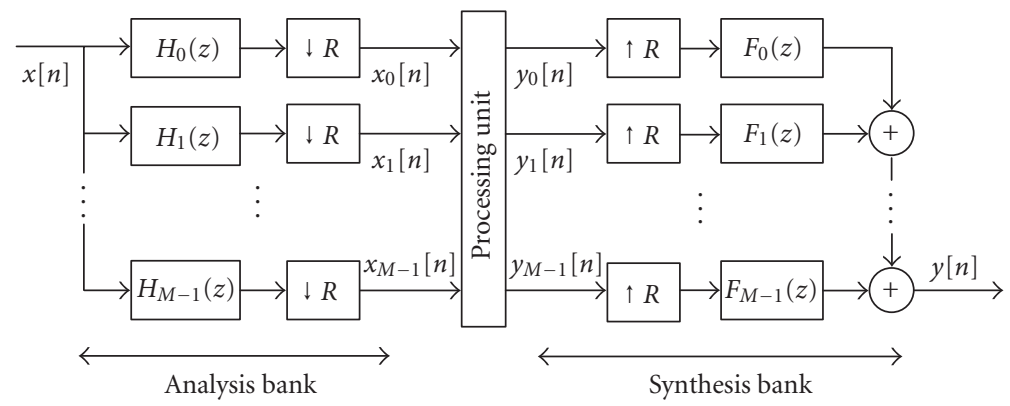

FIGURE 1: $M$-channel filterbank.

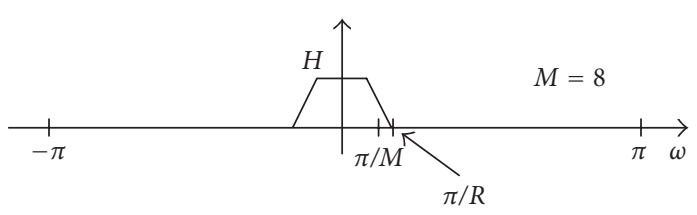

(a)

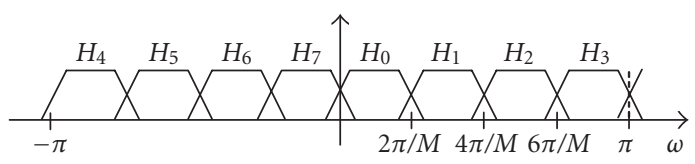

(b)

FIGURE 2: Magnitude response of an eight-channel GDFT filterbank: (a) prototype filter, (b) analysis filters.

in the PR case), whose delay is $D=N_{h}$. Design methods for such FBs are given in $[2,8,9]$ (and $[5,7]$ in the PR case).

In applications where low delay is important, better filtering properties are obtained by using two distinct prototypes in (1); the prototypes are related only by the NPR condition, as detailed later in Section 2. In this case we can take $N_{h}, N_{f}>D$. The method proposed in this paper designs twoprototypes FBs; other algorithms have been given previously in $[1,6,10]$.

The structure of the paper is as follows. In Section 2, we show how the NPR property can be expressed in a simpler way and also give an explicit form of the distortion transfer function for GDFT FBs. In Section 3, we describe our design algorithm with separate treatments of the near-orthogonal and two-prototypes cases. A near-orthogonal FB is designed by solving a semidefinite programming (SDP) problem. For two-prototypes FBs, the analysis and synthesis banks are successively optimized, each time solving a second-order cone programming (SOCP) problem. Both SDP and SOCP are instances of convex programming, for which the solution can be found with reliable algorithms. In Section 4, we give two examples of design and analyze the performance of the FBs as a function of the order of the prototypes; results of some synthetic echo control experiments are also presented. In the remainder of this paper, by FB we actually understand an oversampled FB.

\section{SIMPLIFIED NPR CONDITIONS}

The input-output relation for the FB from Figure 1 is

$$
Y(z)=T_{0}(z) X(z)+\sum_{\ell=1}^{R-1} T_{\ell}(z) X\left(z \mathrm{e}^{-j 2 \pi \ell / R}\right),
$$

where

$$
T_{0}(z)=\frac{1}{R} \sum_{k=0}^{M-1} H_{k}(z) F_{k}(z)
$$

is the distortion transfer function and determines the distortion caused by the overall system for the unaliased component $X(z)$ of the input signal, and

$$
T_{\ell}(z)=\frac{1}{R} \sum_{k=0}^{M-1} H_{k}\left(z \mathrm{e}^{-j 2 \pi \ell / R}\right) F_{k}(z),
$$

for $\ell=1: R-1$ are called aliasing transfer functions and determine how well the aliased components $X\left(z \mathrm{e}^{-j 2 \pi \ell / R}\right)$ of the input signal are attenuated.

A PR FB obeys the conditions $T_{0}(z)=z^{-D}$ and $T_{\ell}(z)=0$, for $\ell=1: R-1$. For NPR FBs, the conditions are relaxed to the following forms.

Condition 1. The frequency response of the distortion transfer function is near the response of a pure delay, that is,

$$
\left|T_{0}\left(\mathrm{e}^{j \omega}\right)-\mathrm{e}^{-j D \omega}\right| \leq \delta_{d}, \quad \forall \omega \in[0,2 \pi],
$$

where $\delta_{d}$ is a preset tolerance.

Condition 2. The frequency responses of the aliasing transfer functions are very small, that is,

$$
\left|T_{\ell}\left(\mathrm{e}^{j \omega}\right)\right| \leq \delta_{a}, \quad \forall \ell=1: R-1, \forall \omega \in[0,2 \pi],
$$

where $\delta_{a}$ is a preset tolerance, normally taken such that $\delta_{a}<$ $\delta_{d}$.

A common way to impose the conditions (5) and (6) is by using a grid of frequencies instead of the whole interval $[0,2 \pi]$. Even so, the implementation of (6) has a high computational cost, due to the large number of constraints. Condition 2 can be reformulated in the following simpler way. 
Condition 3. The prototype filters, $H(z)$ and $F(z)$, have a magnitude response that is very small outside the baseband $[0, \pi / R]$, as suggested in Figure $2(\mathrm{a})$, that is,

$$
\left|H\left(\mathrm{e}^{j \omega}\right)\right| \leq \mu_{h} \delta_{s}, \quad\left|F\left(\mathrm{e}^{j \omega}\right)\right| \leq \mu_{f} \delta_{s}, \quad \text { for } \pi / R \leq \omega \leq \pi .
$$

The scaling factors $\mu_{h}, \mu_{f}$ are equal to the ideal magnitudes in the passband of the frequency responses of the filters $H_{k}(z)$, $F_{k}(z)$, respectively. Without loss of generality, we can consider $\mu_{h} \mu_{f}=R$, as in a PR FB.

Condition (7) has an immediate practical motivation: FBs obeying it have reduced aliasing of the subband signals, which is beneficial to subband adaptive filtering. We evaluate below a conservative estimate of the bound $\delta_{a}$ from (6).

We can assume with good approximation that

$$
\left|H\left(\mathrm{e}^{j \omega}\right)\right| \leq \mu_{h}, \quad\left|F\left(\mathrm{e}^{j \omega}\right)\right| \leq \mu_{f}, \quad \text { for } \omega \in[0, \pi / R] .
$$

We restrict our analysis to the interval $[0,2 \pi / R]$, since it is valid for all other intervals of length $2 \pi / R$. In this interval, the inequality

$$
\left|H_{k}\left(\mathrm{e}^{j(\omega-2 \pi \ell / R)}\right)\right|\left|F_{k}\left(\mathrm{e}^{j \omega}\right)\right| \leq \begin{cases}R \delta_{s}, & \text { if } k \in \mathcal{K}_{\ell} \\ R \delta_{s}^{2}, & \text { otherwise }\end{cases}
$$

holds, where $\mathcal{K}_{\ell}$ is the set of indices $k$ for which either the passband of $\left|F_{k}\left(\mathrm{e}^{j \omega}\right)\right|$ or the passband of $\left|H_{k}\left(\mathrm{e}^{j(\omega-2 \pi \ell / R)}\right)\right|$ has a nonzero intersection with $[0,2 \pi / R]$ (note that the two passbands are disjoint). Neglecting the terms in $\delta_{s}^{2}$, it results from (9) and (4) that

$$
\left|T_{\ell}\left(\mathrm{e}^{j \omega}\right)\right| \leq\left|\mathcal{K}_{\ell}\right| \delta_{s}
$$

where $\left|\mathcal{K}_{\ell}\right|$ is the number of elements of the set $\mathcal{K}_{\ell}$. So, the aliasing transfer functions obey Condition 2 with $\delta_{a} \leq$ $\left|\mathcal{K}_{\ell}\right| \delta_{s}$ in (6). From (7) and (8), it can be proved that

$$
\left|\mathcal{K}_{\ell}\right| \leq \kappa=2\left(\left\lceil\frac{M}{R}\right\rceil-1\right)
$$

Imposing Conditions 1 and 3 leads to the NPR relation

$$
\left|Y\left(\mathrm{e}^{j \omega}\right)-\mathrm{e}^{-j D \omega} X\left(\mathrm{e}^{j \omega}\right)\right| \leq \tilde{\delta}_{d}
$$

where $\tilde{\delta}_{d}>\delta_{d}$ but usually $\tilde{\delta}_{d} \approx \delta_{d}$. We scale the input signal such that $\left|X\left(\mathrm{e}^{j \omega}\right)\right| \leq 1$ and so $\tilde{\delta}_{d}$ can be interpreted as a relative NPR error with respect to the actual peak value of $\left|X\left(\mathrm{e}^{j \omega}\right)\right|$. Taking (10) into account, the input-output relation (2) shows that, in a worst case scenario, we have

$$
\tilde{\delta}_{d} \leq \delta_{d}+\kappa(R-1) \delta_{s}
$$

In a practical setup, even though $\kappa(R-1) \delta_{s}$ might be greater than $\delta_{d}$, an error $\widetilde{\delta}_{d}$ of the same order of magnitude as $\delta_{d}$ is obtained. This is due to the following reasons.

(i) The inequality (8) is too loose. Actually, the frequency responses of the prototype filters are approximately equal to $\mu_{h}$ and $\mu_{f}$ in the passband $[0, \pi / M]$, then decay rapidly in the transition band $[\pi / M, \pi / R]$. Thus, the inequality (10) holds for a bound of $\left|\mathcal{K}_{\ell}\right|$ much smaller than that indicated by (11).

(ii) If the prototype filters are optimized in a least-squares sense, as we consider appropriate, the magnitudes $\left|H\left(\mathrm{e}^{j \omega}\right)\right|$ and $\left|F\left(\mathrm{e}^{j \omega}\right)\right|$ are actually much smaller than $\delta_{s}$ almost everywhere in the interval $[\pi / R, \pi]$. Consequently, for many values of $\ell$, the products from (9) have much lower bounds.

(iii) Partial cancellation of errors at addition may appear in (4) and (2).

We will present in Section 4 some measures and values of the NPR error for FBs designed by respecting Conditions 1 and 3 .

A design principle similar to that exposed above was used for near-orthogonal FBs in $[2,9,11]$ (in the latter paper for nonuniform FBs). Actually, in these papers, Condition 1 had the form of a power complementarity constraint, specific to orthogonal FBs. In our paper, the principle is adapted to the general case of two-prototypes FBs.

Until now, we have simplified Condition 2 by replacing (6) with (7). Further analysis reveals that the distortion transfer function (3) has a very simple form in the case of GDFT FB and so Condition 2 can be implemented with low complexity.

Let $\mathbf{h}, \mathbf{f}$ be the vectors containing the coefficients of the analysis and synthesis prototypes, respectively (of length $N_{h}+$ 1 and $N_{f}+1$, resp.). Then, the distortion transfer function (3) has the expression (see the Appendix for a proof)

$$
T_{0}(z)=\frac{M}{R} \sum_{i, 0 \leq D+i M \leq N_{t}}(-1)^{i}\left(\mathbf{h}^{T} \Psi_{D+i M} \mathbf{f}\right) z^{-(D+i M)},
$$

where $N_{t}=N_{h}+N_{f}$ is the order of the distortion transfer function and $\Psi_{n}$ is the elementary Hankel matrix having ones on the $n$th antidiagonal and zeros elsewhere (antidiagonals are counted from zero, starting from the upper-left corner).

There are at most $\left(N_{t}+1\right) / M$ nonzero coefficients in the sum from (14), that is, a very small number; this is a very favorable feature in reducing the complexity of optimization algorithms using the constraint (5). We are not aware of any algorithm for designing two-prototypes FBs that takes advantage of the form (14).

Let us also note that, for any $0 \leq n \leq N_{t}$, the bilinear form $\mathbf{h}^{T} \boldsymbol{\Psi}_{n} \mathbf{f}$ may be written as the convolution

$$
\mathbf{h}^{T} \Psi_{n} \mathbf{f}=\sum_{m=\max \left(0, n-N_{f}\right)}^{\min \left(n, N_{h}\right)} h[m] f[n-m]
$$




\section{PROPOSED DESIGN ALGORITHMS}

\subsection{Previous work and outline of our contribution}

The best method for designing near-orthogonal GDFT FBs appears to be that from [9]. In its basic formulation, the stopband energy of the prototype is optimized, subject to a timedomain constraint on the coefficients of the distortion transfer function (14). Additional constraints can be put on the minimum stopband attenuation and on the energy on the transition band $[\pi / M, \pi / R]$. The optimization problem has an SDP form. More details are given in Section 3.3.

For two-prototypes GDFT FBs, the methods from $[1,6$, 10] optimize separately the analysis and synthesis prototypes. In [10], each prototype is an eigenfilter with approximately linear phase in the passband and so NPR is obtained indirectly. In [6], the analysis prototype is an equiripple linear phase filter (designed with the Remez algorithm) and the synthesis prototype is optimized using a composite criterion involving the $H_{1}$-norm on the stopband and two terms of the sum defining the distortion transfer function error (5). In [1], the analysis prototype is an approximately linear phase filter and the synthesis prototype is obtained by minimizing a quadratic criterion involving the $\mathrm{H}_{2}$-norm of the NPR error (5) and a measure of the residual aliasing distortion (an approximated $\mathrm{H}_{2}$-norm of the aliasing transfer functions (4)).

Our method starts by designing the prototype $H(z)$ of a near-orthogonal FB, using the basic formulation from [9], but with a frequency domain implementation of (5). Using $H(z)$ as analysis prototype, we design the synthesis prototype $F(z)$ by minimizing its stopband energy subject to the NPR condition (5). Finally, we redesign $H(z)$ using an identical procedure. The main differences with respect to the previous methods consist in the initialization step, in the implementation of (5) as it is (i.e., as a bound on the $H_{\infty}$-norm of the distortion transfer function error), and in simplifications of the NPR conditions. We also use different optimization tools, namely SDP and SOCP.

\subsection{Implementation of the simplified NPR conditions}

We implement Condition 1 by imposing (5) on a discrete set of frequencies denoted by $\Omega$. For simpler implementation, the set is taken as an equidistant grid covering the interval $[0, \pi]$. (There is no need to cover the interval $[\pi, 2 \pi]$, since the GDFT FB has real prototypes.) Using expression (14), the condition (5) may be written in the form

$$
\left\|\frac{M}{R}\left[\begin{array}{l}
\mathbf{h}^{T} \mathbf{C}(\omega) \mathbf{f} \\
\mathbf{h}^{T} \mathbf{S}(\omega) \mathbf{f}
\end{array}\right]-\left[\begin{array}{c}
\cos \omega D \\
\sin \omega D
\end{array}\right]\right\| \leq \delta_{d}, \quad \text { for } \omega \in \Omega,
$$

where

$$
\begin{aligned}
& \mathbf{C}(\omega)=\sum_{i, 0 \leq D+i M \leq N_{t}}(-1)^{i} \boldsymbol{\Psi}_{D+i M} \cos \omega(D+i M), \\
& \mathbf{S}(\omega)=\sum_{i, 0 \leq D+i M \leq N_{t}}(-1)^{i} \Psi_{D+i M} \sin \omega(D+i M),
\end{aligned}
$$

are Hankel matrices, constant for a given frequency $\omega \in \Omega$.
The prototypes of the GDFT FB are optimized by minimizing their stopband energy, which has favorable effects on aliasing and also ensures good filtering properties. The stopband energy (of the analysis prototype) is

$$
E_{s}=\frac{1}{\pi} \int_{\omega_{s}}^{\pi}\left|H\left(\mathrm{e}^{j \omega}\right)\right|^{2} d \omega=\mathbf{h}^{T} \mathbf{\Phi} \mathbf{h},
$$

where $\omega_{s}$ is the stopband edge and $\boldsymbol{\Phi}$ is a positive definite Toeplitz matrix with the element $\phi_{n}$ on diagonal $n$ defined by

$$
\phi_{n}= \begin{cases}1-\frac{\omega_{s}}{\pi}, & \text { if } n=0, \\ -\frac{\sin \left(\omega_{s} n\right)}{\pi n}, & \text { otherwise. }\end{cases}
$$

There are (at least) two possibilities of implementing Condition 3. The first is to impose (7) on a grid of frequencies, for a given tolerance $\delta_{s}$. The minimization of the stopband energy (18) constrained to (7) is a peak-constrained least-squares (PCLS) optimization [12]. The second possibility is to take a stopband edge $\omega_{s}$ smaller than $\pi / R$, namely

$$
\omega_{s}=\frac{(1+\rho) \pi}{M}
$$

with $\rho$ smaller than $M / R-1$. In this case, the minimization of (7) gives as byproduct a stopband ripple $\delta_{s}$ to satisfy condition (7). (In least-squares optimization, the worst stopband attenuation is typically obtained at the stopband edge; taking $\omega_{s}<\pi / R$ has the effect of decreasing the largest ripple for $\omega \in[\pi / R, \pi]$, while the energy in the same interval also increases.) Although the first method allows complete control on $\delta_{s}$, we prefer the second as having a lighter implementation. The choice of $\rho$ depends on $M / R$ and on the subband processing application. Some examples will be given in Section 4.2. As a general rule, the smaller is $M / R$, the nearer $\rho$ should be to $M / R-1$. It is also possible to use different stopband edges for the analysis and synthesis prototypes, as argued in [10]; in this case, we denote $\rho_{h}$ and $\rho_{f}$, respectively, the factors from (20).

The GDFT FB could be optimized by minimizing a (possibly weighted) sum of the stopband energies of the analysis and synthesis prototypes, subject to the NPR constraints (16). Although the optimization criterion is convex, the constraints (16) are bilinear, which makes the entire optimization problem nonconvex; avoiding local minima is a wellknown difficulty. We propose a different approach described in the sequel and based on convex optimization with standard algorithms (SDP, SOCP).

\subsection{Design of near-orthogonal FBs}

We present here the basic idea from [9], with some modifications in the form of the NPR constraint. In a near-orthogonal $\mathrm{FB}$, the synthesis prototype vector $\mathbf{f}$ contains the coefficients of the analysis prototype $\mathbf{h}$ in reverse order, that is, $f[n]=$ $h\left[N_{h}-n\right]$. Then, the next equality holds:

$$
\Psi_{n} \mathbf{f}=\boldsymbol{\Theta}_{n} \mathbf{h},
$$


Input:

$M$ : the number of channels,

$D$ : FB delay,

$R$ : the down-sampling factor,

$\omega_{s}$ : the stopband edge,

$\delta_{d}$ : the maximum NPR error from (5),

$N_{h}, N_{f}$ : the orders of the analysis and synthesis prototypes,

$N_{0}$ : the order of the analysis prototype for the first iteration,

$\Omega$ : a grid of frequencies covering the interval $[0, \pi]$.

(1) Design oversampled NPR near-orthogonal FB, with prototype $H(z)$ of order $N_{0}$, by solving the SDP problem (22).

(2) Solve the SOCP problem (22) for $F(z)$ of order $N_{f}$, using $H(z)$ computed at Step 1.

(3) Reversing the roles of $\mathbf{h}$ and $\mathbf{f}$, solve the SOCP problem (24) for $H(z)$ of order $N_{h}$, using $F(z)$ computed at Step 2.

Algorithm 1: Outline of the proposed algorithm for designing the prototype filters of an oversampled NPR biorthogonal filterbank.

where $\Theta_{n}$ is the elementary Toeplitz matrix with ones on the $n$th diagonal and zeros elsewhere. The problem of minimizing the energy (18) subject to the NPR constraints (16) becomes

$$
\begin{gathered}
\min _{h} \mathbf{h}^{T} \mathbf{\Phi} \mathbf{h} \\
\text { s.t. }\left\|\frac{M}{R}\left[\begin{array}{c}
\mathbf{h}^{T} \tilde{\mathbf{C}}(\omega) \mathbf{h} \\
\mathbf{h}^{T} \tilde{\mathbf{S}}(\omega) \mathbf{h}
\end{array}\right]-\left[\begin{array}{c}
\cos \omega D \\
\sin \omega D
\end{array}\right]\right\| \leq \delta_{d}, \quad \text { for } \omega \in \Omega,
\end{gathered}
$$

where the Toeplitz matrices $\widetilde{\mathbf{C}}(\omega)$ and $\widetilde{\mathbf{S}}(\omega)$ are defined as in (17) but with $\boldsymbol{\Theta}_{D+i M}$ replacing $\boldsymbol{\Psi}_{D+i M}$. Problem (22) is equivalent to a convex optimization problem, in terms of the coefficients of the product filter $H(z) H\left(z^{-1}\right)$, which have the form $\mathbf{h}^{T} \Theta_{n} \mathbf{h}, n=-N_{h}: N_{h}$; see $[13,14]$ for details on this type of transformation, that leads to a semidefinite programming problem. The transformation of (22) into a convex problem is possible also due to the Toeplitz structure of the matrices appearing there, as explained in [15]. Each frequency domain constraint of type (16) has a second order cone form; SOCP is a particular case of SDP, with faster implementation.

\subsection{Design of two-prototypes FBs}

Let us assume for the beginning that the analysis prototype $H(z)$ is given. We next show that the optimization of the synthesis prototype has a convex formulation, as a SOCP problem. Minimizing the stopband energy of $F(z)$ subject to the NPR conditions (16), that is,

$$
\begin{gathered}
\min _{f} \mathbf{f}^{T} \mathbf{\Phi f} \\
\text { s.t. }\left\|\frac{M}{R}\left[\begin{array}{l}
\mathbf{h}^{T} \mathbf{C}(\omega) \mathbf{f} \\
\mathbf{h}^{T} \mathbf{S}(\omega) \mathbf{f}
\end{array}\right]-\left[\begin{array}{c}
\cos \omega D \\
\sin \omega D
\end{array}\right]\right\| \leq \delta_{d}, \quad \text { for } \omega \in \Omega,
\end{gathered}
$$

can be expressed in the form

$$
\begin{gathered}
\min _{f, \alpha} \alpha \\
\text { s.t. } \quad\left\|\boldsymbol{\Phi}^{1 / 2} \mathbf{f}\right\| \leq \alpha, \\
\left\|\frac{M}{R}\left[\begin{array}{l}
\mathbf{c}^{T}(\omega) \\
\mathbf{s}^{T}(\omega)
\end{array}\right] \mathbf{f}-\left[\begin{array}{c}
\cos \omega D \\
\sin \omega D
\end{array}\right]\right\| \leq \delta_{d}, \quad \text { for } \omega \in \Omega,
\end{gathered}
$$

where $\mathbf{c}(\omega)=\mathbf{C}(\omega) \mathbf{h}$ and $\mathbf{s}(\omega)=\mathbf{S}(\omega) \mathbf{h}$ are constant vectors for a given frequency $\omega$. The optimization problem (24) is a typical SOCP problem.

Certainly, if $F(z)$ is given, then $H(z)$ can be optimized similarly. We propose the following idea for the design of a two-prototypes FB. First, a near-orthogonal FB is designed, using the algorithm suggested in Section 3.3, namely by solving the problem (22). The obtained prototype $H(z)$ is used as analysis prototype and a synthesis prototype $F(z)$ is computed by solving the SOCP problem (24). Finally, a new analysis prototype is obtained solving the problem similar to (24) for given $F(z)$ and unknown $H(z)$. The algorithm is described formally in Algorithm 1 above. We note that the initial prototype (of the near-orthogonal FB) may have an order $N_{0}$ different from $N_{h}$; in our experiments, we have remarked that it is beneficial to take $N_{0} \leq N_{h}, N_{0} \leq N_{f}$. Our algorithm imposes no a priori relation between the delay $D$ and the orders $N_{h}, N_{f}$.

The steps 2 and 3 of Algorithm 1 can be iterated. Denoting $E_{h}$ and $E_{f}$ the stopband energies of the analysis and synthesis prototypes, respectively, it is easy to see that successive iterations decrease (or keep constant) the values of $E_{h}$ and $E_{f}$. If $\mathbf{h}$ and $\mathbf{f}$ are the results of the previous iteration, then they satisfy the NPR condition (16). Thus, minimizing $E_{h}$ subject to (16) for the given $\mathbf{f}$, as in Step 2 of the algorithm, cannot produce a worse value of $E_{h}$ in the current iteration. Similarly, the values of $E_{f}$ form a nonincreasing sequence.

However, the iterations usually do not bring significant improvement. The improvement appears especially when there is a big disparity between $E_{h}$ and $E_{f}$ after the first iteration; further iterations mostly decrease the largest of $E_{h}$ and 
$E_{f}$. Sometimes, the stopband energy reduction may come together with an unwanted increase of the passband ripple. Moreover, in the synthetic echo control experiments described in Section 4.2, we have noticed only very small differences between the FBs optimized as in Algorithm 1 and those optimized with more iterations. For these reasons, we will not report results for the iterative algorithm.

\section{EXPERIMENTAL RESULTS}

The algorithms described in the previous section have been implemented using Matlab and the SDP library SeDuMi [16] (which includes algorithms for SOCP problems). The programs have been run on a Pentium III PC at $1 \mathrm{GHz}$. In this section, we give two examples of design, some curves showing the dependence of the stopband energy and attenuation as functions of the orders $N_{h}, N_{f}$, and also results that are obtained in a synthetic echo control experiment. All filterbanks designed and used here have $M=64$ channels; since real signals are processed, only 32 out of these 64 channels are necessary. The delay is $D=80$. In all designs, a grid $\Omega$ of 100 points was used. The other parameters of Algorithm 1, which may differ from one design to another, will be specified when appropriate. In particular, the down-sampling factor $R$ takes values between 16 and 24 and filter orders between 80 and 180 . The oversampling ratios $M / R$ are relatively high, but this is due to the small values of the orders with respect to the number of channels (i.e., the ratios $N_{h} / M$ are relatively small).

\subsection{Examples of design}

Example 1. The down-sampling factor $R$ is 16. Accordingly, the stopband edge $\omega_{s}$ is defined by $\rho=2.9$ (instead of $M / R-1=3)$. The distortion transfer function bound is $\delta_{d}=0.003$ (about $-50 \mathrm{~dB}$ ). We want prototypes that have at least $60 \mathrm{~dB}$ stopband attenuation (i.e., $\delta_{s}=0.001$ in (7)). To obtain such prototypes, we have run our algorithm for different orders $N_{0}, N_{h}$, and $N_{f}$; the search is limited by considering only prototypes with similar orders $\left(\left|N_{h}-N_{f}\right| \leq 4\right)$. We report the FBs with smallest order satisfying the requirements. We have obtained $N_{h}=96, N_{f}=94$ (with $N_{0}=76$ ). The frequency responses of the analysis and synthesis prototypes are shown in Figure 3. The attenuations outside the baseband are at least $A_{h}=60.5 \mathrm{~dB}$ and $A_{f}=60.0 \mathrm{~dB}$.

Next, we analyze the NPR properties of the FB. As the distortion transfer function error from (5) is bounded by the chosen $\delta_{d}$, we try to evaluate the contribution of the aliasing terms. To this purpose, we first compute the a posteriori worst case bound that aliasing transfer functions can attain in (2), namely

$$
T_{\max }\left(\mathrm{e}^{j \omega}\right)=\sum_{\ell=1}^{R-1}\left|T_{\ell}\left(\mathrm{e}^{j \omega}\right)\right| .
$$

For the designed FB, we obtain the bound

$$
\delta_{\max }=\max _{\omega} T_{\max }\left(\mathrm{e}^{j \omega}\right) \approx 0.0028,
$$

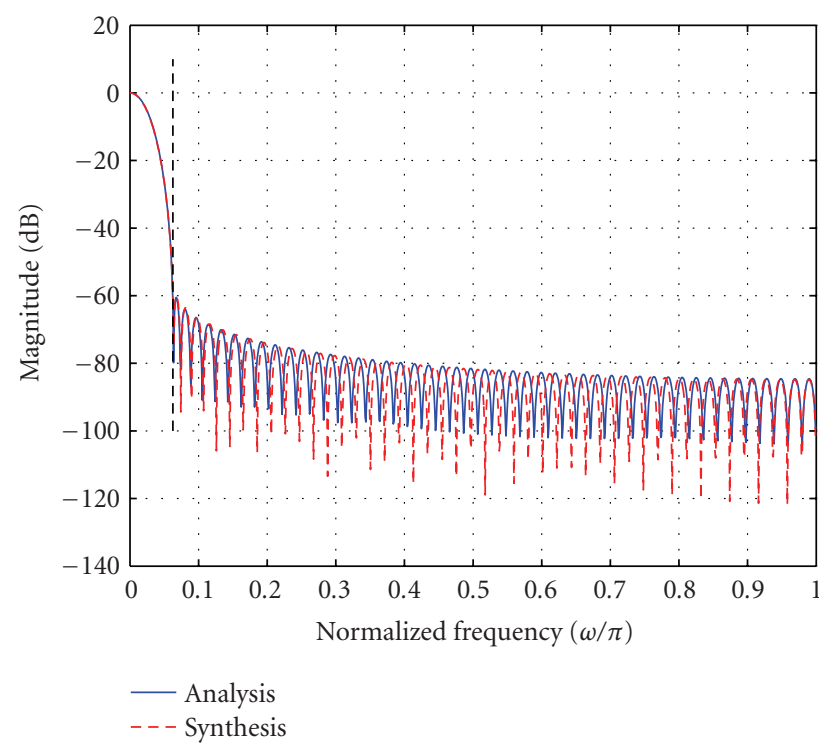

FIgURE 3: Frequency responses of prototypes in Example 1.

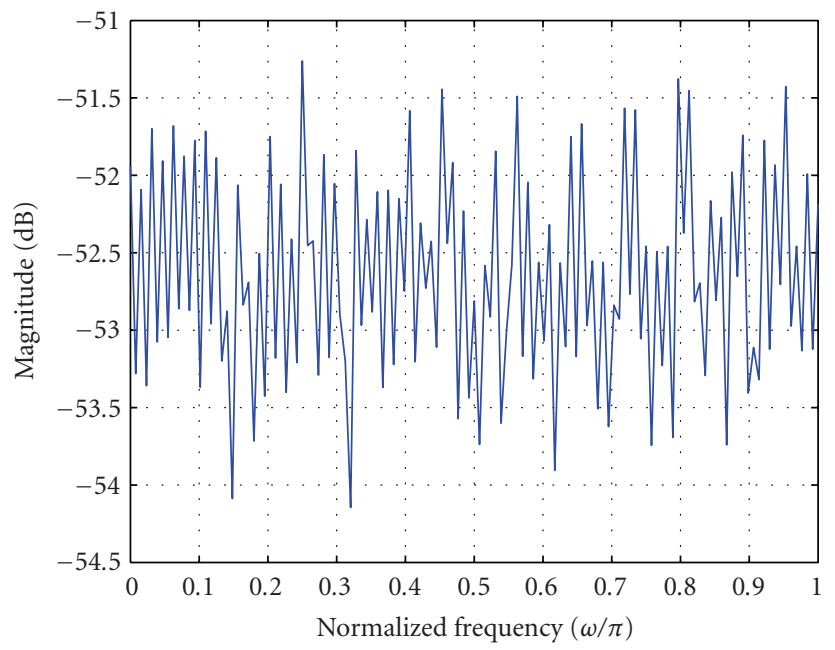

FIGURE 4: Power spectral density of reconstruction error for the FB in Example 1.

that is, a value of the same order as $\delta_{d}$. Assuming an input signal with $\left|X\left(\mathrm{e}^{j \omega}\right)\right| \leq 1$, the NPR error (12) may be bounded by

$$
\widetilde{\delta}_{d} \leq \delta_{d}+\delta_{\max }
$$

To evaluate the true value of $\tilde{\delta}_{d}$, we have simulated the behavior of the designed FB for a Gaussian input signal $x[n]$ (generated with the function randn in Matlab) with 32000 samples. The power spectral density of the reconstruction error $e[n]=y[n]-x[n-D]$ is shown in Figure 4; it is visible that the reconstruction error is below $50 \mathrm{~dB}$, that is, the bound imposed in the specification only for the distortion transfer function error; so, we have obtained $\tilde{\delta}_{d} \approx \delta_{d}$. 
TABle 1: Attenuations and stopband energies for prototypes obtained with different initial orders $N_{0}$ (with $N_{h}=96, N_{f}=94$ ).

\begin{tabular}{c|cccccc}
\hline$N_{0}$ & $A_{h}$ & $A_{f}$ & $\left(A_{h}+A_{f}\right) / 2$ & $E_{h}$ & $E_{f}$ & $\left(E_{h}+E_{f}\right) / 2$ \\
\hline 64 & 58.37 & 63.10 & 60.73 & $3.1 \mathrm{e}-8$ & $0.7 \mathrm{e}-8$ & $1.93 \mathrm{e}-8$ \\
68 & 59.24 & 60.88 & 60.06 & $2.4 \mathrm{e}-8$ & $1.3 \mathrm{e}-8$ & $1.89 \mathrm{e}-8$ \\
72 & 59.99 & 60.45 & 60.22 & $2.0 \mathrm{e}-8$ & $1.6 \mathrm{e}-8$ & $1.81 \mathrm{e}-8$ \\
76 & 60.50 & 60.02 & 60.26 & $1.8 \mathrm{e}-8$ & $1.9 \mathrm{e}-8$ & $1.81 \mathrm{e}-8$ \\
80 & 60.72 & 59.88 & 60.30 & $1.6 \mathrm{e}-8$ & $2.0 \mathrm{e}-8$ & $1.82 \mathrm{e}-8$ \\
84 & 60.65 & 59.93 & 60.29 & $1.7 \mathrm{e}-8$ & $1.9 \mathrm{e}-8$ & $1.82 \mathrm{e}-8$ \\
88 & 60.72 & 59.87 & 60.30 & $1.6 \mathrm{e}-8$ & $2.0 \mathrm{e}-8$ & $1.82 \mathrm{e}-8$ \\
92 & 60.86 & 59.73 & 60.30 & $1.6 \mathrm{e}-8$ & $2.1 \mathrm{e}-8$ & $1.84 \mathrm{e}-8$ \\
\hline
\end{tabular}

As a final discussion on Example 1, let us remark that the initial order $N_{0}$ is not a critical parameter of Algorithm 1 . We show in Table 1 the values of the attenuations $A_{h}$ and $A_{f}$ and also of the stopband energies $E_{h}$ and $E_{f}$ (for the analysis and synthesis prototypes, resp.), as well as their averages, for $N_{h}=96, N_{f}=94$ and different values of $N_{0}$. It is clear that the averages are approximately constant; hence, the parameter $N_{0}$ is interesting only if one desires to match the performances of the two prototypes. The numbers in Table 1 suggest also how to modify $N_{0}$; if, after running Algorithm 1 with a certain $N_{0}$, we decide to improve $H(z)$, then the algorithm has to rerun with a larger value of $N_{0}$; most likely, the new $F(z)$ will have worse stopband energy than in the first run; if, on the contrary, we want to improve $F(z)$ at the expense of $H(z)$, we decrease $N_{0}$. Practical experience showed that few runs of the algorithm are enough to produce the desired result. The design time for each such FB is about 6 seconds.

Example 2. The only parameters changing values with respect to Example 1 are $R=20$ and $\rho=2.1$ (while $M / R-1=$ 2.2). The frequency responses of the prototypes are shown in Figure 5. The orders are $N_{h}=130$ and $N_{f}=134$ (with $N_{0}=$ 124). The attenuations are $A_{h}=61.0 \mathrm{~dB}$ and $A_{f}=61.3 \mathrm{~dB}$, and the stopband energies are $E_{h}=1.17 \mathrm{e}-8$ and $E_{f}=5.72 \mathrm{e}-$ 8. We see that matching the attenuations of the analysis and synthesis prototypes is made at the expense of a difference in the stopband energies. The execution time for obtaining the $\mathrm{FB}$ is about 17 seconds.

Attainable stopband energies and attenuations. We now present some more general results given by our algorithm for different orders of the prototype filters. There are three sets of specifications, the difference being made by the downsampling factor $R$, which takes the values 16, 20, and 24; accordingly, the stopband edge parameter $\rho$ has the values 2.9, 2.1, and 1.6, respectively. (Particular cases of the first two sets of specifications produced Examples 1 and 2.) The tolerance $\delta_{d}$ is $0.003,0.003$, and 0.01 , respectively (the last value is higher due to the worse attenuations obtained). The order $N_{f}$ takes values from 80 to 180 and $N_{h}$ is taken such that $\left|N_{h}-N_{f}\right| \leq 4$. The initialization order $N_{0}$ takes values

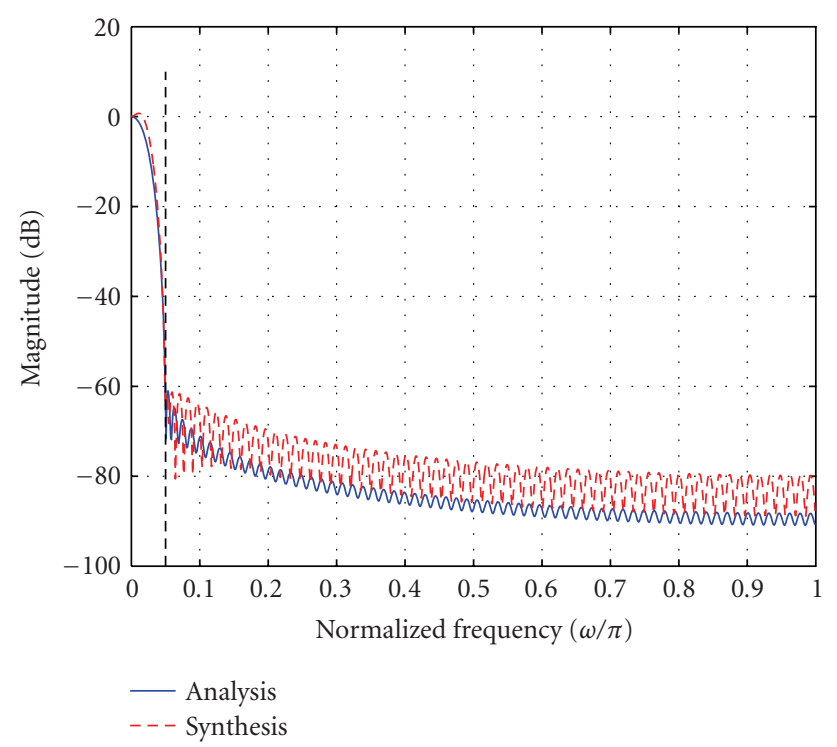

FIGURE 5: Frequency responses of prototypes in Example 2.

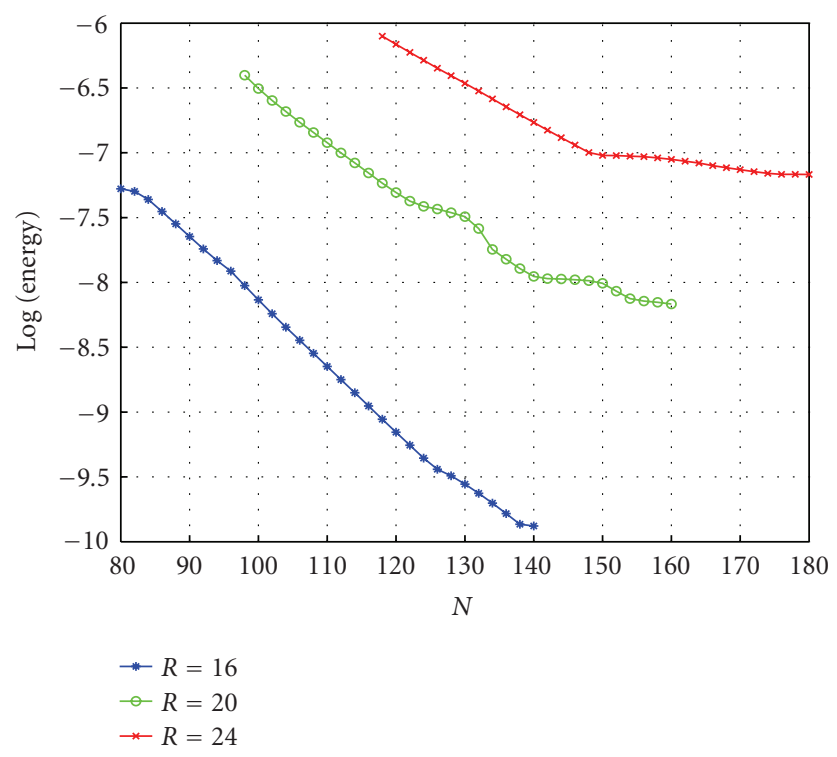

FIGURE 6: Stopband energies of prototypes of GDFT FB, function of order and downsampling factor $R$.

between $N_{f}-40$ and $N_{f}$. Figure 6 presents values of the average stopband energy $\left(E_{h}+E_{f}\right) / 2$, function of the "average order" $\left(N_{h}+N_{f}\right) / 2$ of the FB. For each average order, there are many FB designed, with different values of $N_{0}, N_{h}$, and $N_{f}$; the best average stopband energy is used in the graph; however, as suggested in Table 1, there are several initializations that give similar results. In Figure 7, the average attenuation $\left(A_{h}+A_{f}\right) / 2$ is presented. As the stopband energy is optimized in our algorithm, its values decrease as the order increases. The attenuations follow the same trend, although with some 


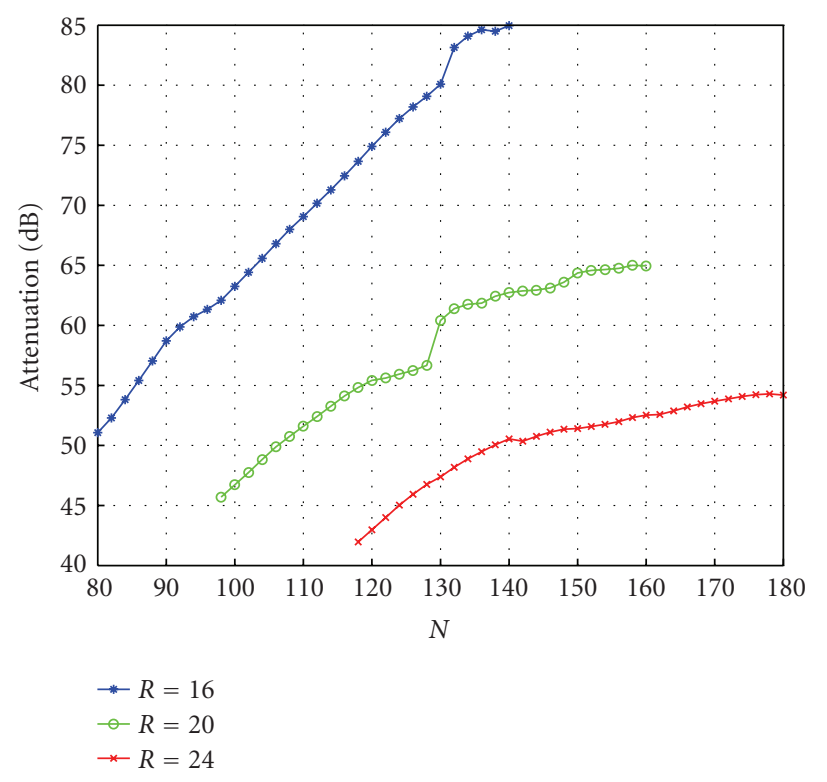

FIGURE 7: Attenuation of prototypes of GDFT FB, function of order and downsampling factor $R$.

small irregularities. The results in Figures 6 and 7 show that our method can be used for a large domain of specifications and thus for a large range of applications.

Finally, we mention that we have tested our programs for orders up to 250 (when the execution time is 200-220 seconds).

\subsection{Synthetic echo control experiments}

We have studied the behavior of the FBs designed with our method in the subband adaptive filtering scheme from Figure 8. The experimental setup is similar to that from [9]. The reference signal $r[n]$ is obtained by passing the white noise $x[n]$ through the "echo path" $C(z)$, which is a length 64 FIR filter whose coefficients are $c[n]=u[n] e^{-n / 10}$, where $u[n]$ is a zero-mean iid Gaussian signal (generated with randn in Matlab). The dashed box describes only processing on channel $k$, similar schemes being employed on the other channels. The adaptive filters $W_{k}(z)$ have length 4 ; their coefficients are adapted following the NLMS algorithm [17, page $324]$, with step size $\tilde{\mu}=0.8$. To study only the performance of the FB, we take $v[n]=0$, that is, there is no noise added to the reference signal.

Table 2 presents the values of the steady-state mean square error (SS-MSE) for orders of the FB between 80 and 130 and three down-sampling factors $(R=24,20,16)$; each SS-MSE is an average over 100 realization of $C(z)$ and the input signal (the same for all experiments). For each subband adaptive filtering experiment, we have performed an ad hoc optimization of the parameters of the design algorithm from Algorithm 1, as follows. The orders $N_{h}$ and $N_{f}$ are taken equal in order to reduce the number of optimization variables. For each $N_{h}$, the parameters $\delta_{d}, N_{0}$, and $\rho$ are given values and the SS-MSE is computed for a very small number of echo paths. Then, the parameters are changed one by one, with a rather coarse step. The value of the NPR bound $\delta_{d}$ is set to a value that results in the optimization of near-orthogonal FBs (when $N_{h}=D=80$ ) and then kept constant for larger $N_{h}$. We start the optimization with $N_{0}=N_{h}-20$ and $\rho=M / R-1$, then decrease $\rho$ until no more improvements in the SS-MSE are obtained. Finally, $N_{0}$ is varied with steps of 5 . These guidelines have been applied with more care for smaller values of $N_{h}$; for large values, the "knowledge" accumulated in the optimization process has been used.

Although we cannot claim that the designed FBs are optimal in terms of achieved SS-MSE, the obtained results support the conclusion that, when the delay is fixed, the performance (here the SS-MSE) can be improved significantly by increasing the order of the FB. In Table 2, the two columns with $N_{h}=80$ correspond to near-orthogonal designs; in the first, the stopband edge parameter from (20) is $\rho=M / R-1$, this is the basic design from [9]; in the second, $\rho$ is decreased to its optimal value (excepting the case $R=24$ ); reducing $\rho$ has the effect of reducing the minimum stopband attenuation and the energy in the transition band $[\pi / M, \pi / R]$ of the prototype, which in [9] are bounded exactly. As in [9], for small $M / R$, the basic design is near-optimal. The other columns of Table 2 show values of SS-MSE obtained with two-prototypes FBs. It appears that the larger $N_{h}$ is, the smaller the values of $\rho$ are better. The improvement in terms of SS-MSE may be more than $4 \mathrm{~dB}$ with respect to the optimized near-orthogonal FB. Moreover, the improvement is significant also when $M / R$ is relatively small.

Another series of experiments have been dedicated to FBs with different stopband edges of the analysis and synthesis prototypes. In this case, we have found that it is better to change the roles of $H(z)$ and $F(z)$ in Algorithm 1. The results are shown in Table 3 only for $R=16$ and $R=20$; as for $R=24$ the improvements are either inexistent or not significant. Contrary to the recommendations of [10], we have obtained better results (in terms of SS-MSE) when the passband of the analysis prototype is narrower than that of the synthesis prototype. However, the improvements with respect to the case $\rho_{h}=\rho_{f}$ are rather small, the maximum being $0.6 \mathrm{~dB}$ for $R=20$ and $N_{h}=130$.

The improvement in SS-MSE comes together with a somewhat slower convergence. The top two curves in Figure 9 represent the MSE for the two extreme cases for $R=20$, namely the near-orthogonal FB with $N_{h}=80$ and SS-MSE $=-30.37 \mathrm{~dB}$ from Table 2 and the two-prototypes FB with $N_{h}=130$ and SS-MSE $=-35.22 \mathrm{~dB}$ from Table 3.

For comparison, we have also designed FBs with $N_{h}=$ 130 , but relaxing the delay constraint to $D=130$. The SSMSE of the best such two-prototypes FB is $-36.21 \mathrm{~dB}$, that is, about $1 \mathrm{~dB}$ better than that of the best FB with $D=80$; its MSE is the bottom one (and the best) in Figure 9. Somewhat surprisingly, the best near-orthogonal FB with $N_{h}=D=130$ gave similar performance to that of the best two-prototypes FB with $D=80$; its MSE is barely distinguishable from the middle curve from Figure 9 and so it was not represented there. 


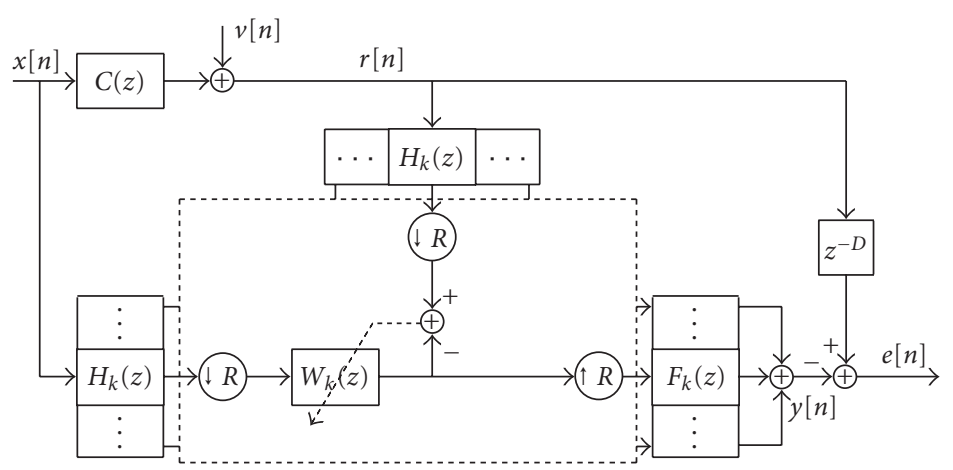

Figure 8: Subband echo control structure.

TABLE 2: SS MSE values (in dB) obtained in the echo control experiment and the optimal values of the FB design parameter $\rho$.

\begin{tabular}{l|c|ccrrrrr}
\hline & $N_{h}, N_{f}$ & 80 & 80 & 90 & 100 & 110 & 120 & 130 \\
\hline$R=24$ & SS MSE & -27.02 & -27.01 & -26.96 & -28.64 & -29.67 & -30.19 & -30.41 \\
$\delta_{d}=0.008$ & $\rho$ & 1.666 & 1.65 & 1.65 & 1.6 & 1.6 & 1.5 & 1.5 \\
\hline$R=20$ & SS MSE & -30.19 & -30.37 & -31.25 & -32.26 & -33.34 & -34.37 & -34.62 \\
$\delta_{d}=0.005$ & $\rho$ & 2.2 & 1.95 & 1.9 & 1.9 & 1.85 & 1.6 & 1.55 \\
\hline$R=16$ & SS MSE & -34.31 & -35.24 & -36.39 & -37.40 & -38.37 & -39.24 & -39.59 \\
$\delta_{d}=0.003$ & $\rho$ & 3.0 & 2.2 & 2.1 & 2.1 & 2.0 & 1.8 & 1.7 \\
\hline
\end{tabular}

TABLE 3: SS MSE values (in dB) and the optimal values of the design parameter $\rho_{h}, \rho_{f}$.

\begin{tabular}{|c|c|c|c|c|c|c|}
\hline & $N_{h}, N_{f}$ & 90 & 100 & 110 & 120 & 130 \\
\hline$R=20$ & SS MSE & -31.13 & -32.58 & -33.81 & -34.77 & -35.22 \\
\hline \multirow{2}{*}{$\delta_{d}=0.005$} & $\rho_{h}$ & 1.85 & 1.8 & 1.65 & 1.5 & 1.45 \\
\hline & $\rho_{f}$ & 2.1 & 2.1 & 2.1 & 2.1 & 2.1 \\
\hline$R=16$ & SS MSE & -36.46 & -38.06 & -38.93 & -39.60 & -39.79 \\
\hline \multirow{2}{*}{$\delta_{d}=0.003$} & $\rho_{h}$ & 2.0 & 1.9 & 1.9 & 1.7 & 1.6 \\
\hline & $\rho_{f}$ & 2.9 & 2.9 & 2.9 & 2.6 & 2.4 \\
\hline
\end{tabular}

The curves in Figure 9 suggest what can be gained and what is the price when increasing the order and keeping the delay fixed. This kind of tradeoff between order and delay is specific to real-time applications, where the delay is typically constrained to low values; our algorithm allows the design of FBs for such applications. In our echo control experiments, the SS-MSE can be clearly increased by increasing the order, while the convergence rate appears not to be fully controlled by the parameters of our algorithm; the influence of the properties of the prototype filters on the convergence rate has to be further investigated and conveniently expressed into optimization criteria.

\section{CONCLUSIONS}

We have proposed an algorithm for the design of oversampled NPR GDFT modulated filterbanks. The NPR property is achieved via a simplified set of conditions. The algorithm starts by solving the SDP problem (22) for designing the prototype of a near-orthogonal FB, used as initialization.
Then, two SOCP problems (24) are solved, for obtaining first the synthesis, then the analysis prototype; each time, the other prototype is the one given by the solution of the previous problem. With this algorithm, it is possible to design two-prototypes FBs that, for a given delay, have significantly better stopband energy and attenuation than nearorthogonal FBs. An echo control experiment shows the same type of improvement in terms of achieved SS-MSE.

Further work will be dedicated to study methods for designing nonuniform low-delay oversampled FBs.

\section{APPENDIX \\ PROOF OF (14)}

The transfer function of the analysis prototype is expressible as

$$
H(z)=\left[1 z^{-1} \cdots z^{-N_{h}}\right] \cdot \mathbf{h} .
$$




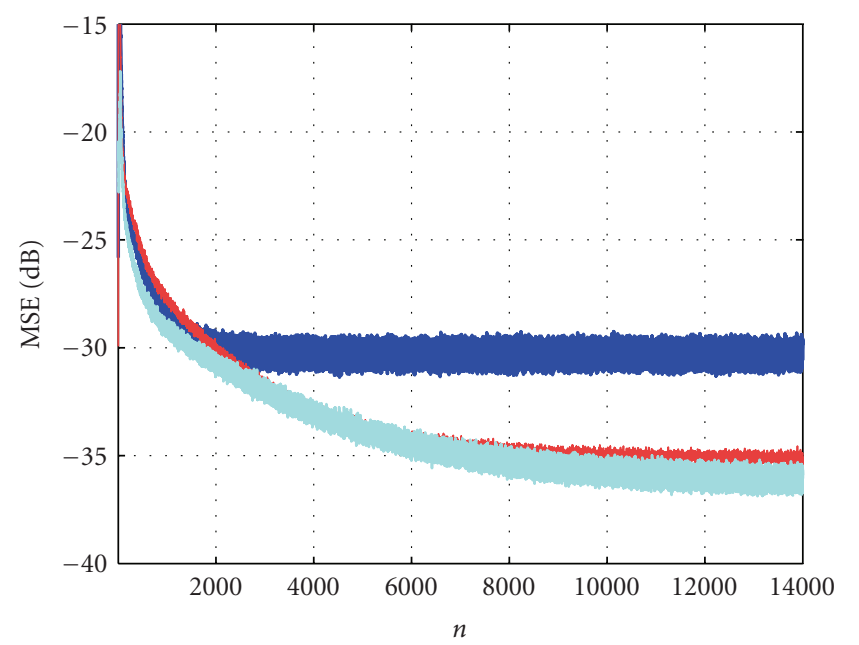

$$
\begin{aligned}
-N_{h} & =80, D=80 \\
N_{h} & =130, D=80 \\
N_{h} & =130, D=130
\end{aligned}
$$

FIgURE 9: MSE (over 1000 realizations of the input signal and the echo path) for $R=20$ and three FBs: near-orthogonal with $N_{h}=$ $D=80$, two-prototypes with $N_{h}=130, D=80$, and two-prototypes with $N_{h}=D=130$.

A similar expression can be given for $F(z)$. Taking into account the modulation expressions (1), a term of the distortion transfer function (3) can be written as

$$
\begin{aligned}
H_{k}(z) F_{k}(z)= & \mathbf{f}^{T}\left[\begin{array}{c}
\vdots \\
\mathrm{e}^{j \pi(2 k+1)(n-D / 2) / M} z^{-n} \\
\vdots
\end{array}\right] \\
& \times\left[\ldots \mathrm{e}^{j \pi(2 k+1)(m-D / 2) / M} z^{-m}\right. \\
& \ldots . \mathbf{h} \\
= & \mathbf{f}^{T} \boldsymbol{\Gamma}_{k}(z) \mathbf{h},
\end{aligned}
$$

where $\Gamma_{k}(z)$ is the Hankel matrix,

$$
\boldsymbol{\Gamma}_{k}(z)=\sum_{n=0}^{N_{t}} \mathrm{e}^{j \pi(2 k+1)(n-D) / M} \boldsymbol{\Psi}_{n} z^{-n} .
$$

Using (A.2) and (A.3), the distortion transfer function (3) becomes

$$
\begin{aligned}
T_{0}(z) & =\frac{1}{R} \mathbf{f}^{T}\left(\sum_{n=0}^{N_{t}} \sum_{k=0}^{M-1} \mathrm{e}^{j \pi(2 k+1)(n-D) / M} \Psi_{n} z^{-n}\right) \mathbf{h} \\
& =\frac{1}{R} \mathbf{f}^{T}\left(\sum_{n=0}^{N_{t}} \mathrm{e}^{j \pi(n-D) / M} \Psi_{n} z^{-n} \sum_{k=0}^{M-1} \mathrm{e}^{j 2 \pi k(n-D) / M}\right) \mathbf{h} .
\end{aligned}
$$

Since

$$
\sum_{k=0}^{M-1} \mathrm{e}^{j 2 \pi k(n-D) / M}= \begin{cases}M, & \text { if }(n-D) \bmod M=0 \\ 0, & \text { otherwise }\end{cases}
$$

and $\mathrm{e}^{j \pi(n-D) / M}=(-1)^{(n-D) / M}$ when $(n-D) \bmod M=0$, the distortion transfer function as given by (A.4) can be written as (14).

\section{ACKNOWLEDGMENTS}

This work is supported by Nokia Research Center, Tampere, Finland. A short version of this paper has been presented at the ICASSP 2005 conference. Prof. Dumitrescu is on leave from the Department of Automatic Control and Computers, "Politehnica" University of Bucharest, Romania.

\section{REFERENCES}

[1] J. M. de Haan, N. Grbic, I. Claesson, and S. E. Nordholm, "Filter bank design for subband adaptive microphone arrays," IEEE Transactions On Speech and Audio Processing, vol. 11, no. 1, pp. 14-23, 2003.

[2] M. Harteneck, S. Weiss, and R. W. Stewart, "Design of near perfect reconstruction oversampled filter banks for subband adaptive filters," IEEE Transactions on Circuits And SystemsPart II: Analog and Digital Signal Processing, vol. 46, no. 8, pp. 1081-1085, 1999.

[3] J. P. Reilly, M. R. Wilbur, M. Seibert, and N. Ahmadvand, "The complex subband decomposition and its application to the decimation of large adaptive filtering problems," IEEE Transactions on Signal Processing, vol. 50, no. 11, pp. 2730-2743, 2002.

[4] S. Weiss, A. Stenger, R. W. Stewart, and R. Rabenstein, "Steadystate performance limitations of subband adaptive filters," IEEE Transactions on Signal Processing, vol. 49, no. 9, pp. 19821991, 2001.

[5] H. Bölcskei and F. Hlawatsch, "Oversampled cosine modulated filter banks with perfect reconstruction," IEEE Transactions on Circuits And Systems - Part II: Analog and Digital Signal Processing, vol. 45, no. 8, pp. 1057-1071, 1998.

[6] K. Eneman and M. Moonen, "DFT modulated filter bank design for oversampled subband systems," Signal Processing, vol. 81, no. 9, pp. 1947-1973, 2001.

[7] J. Kliewer and A. Mertins, "Oversampled cosine-modulated filter banks with arbitrary system delay," IEEE Transactions on Signal Processing, vol. 46, no. 4, pp. 941-955, 1998.

[8] Y.-P. Lin and P. P. Vaidyanathan, "A Kaiser window approach for the design of prototype filters of cosine modulated filterbanks," IEEE Signal Processing Letters, vol. 5, no. 6, pp. 132134, 1998.

[9] M. R. Wilbur, T. N. Davidson, and J. P. Reilly, "Efficient design of oversampled NPR GDFT filterbanks," IEEE Transactions on Signal Processing, vol. 52, no. 7, pp. 1947-1963, 2004.

[10] B. Farhang-Boroujeny and Z. Wang, "Adaptive filtering in subbands: Design issues and experimental results for acoustic echo cancellation," Signal Processing, vol. 61, no. 3, pp. 213223, 1997.

[11] Z. Cvetkovic and J. D. Johnston, "Nonuniform oversampled filter banks for audio signal processing," IEEE Transactions On Speech and Audio Processing, vol. 11, no. 5, pp. 393-399, 2003.

[12] J. W. Adams and J. L. Sullivan, "Peak-constrained least-squares optimization," IEEE Transactions on Signal Processing, vol. 46, no. 2, pp. 306-321, 1998.

[13] B. Alkire and L. Vandenberghe, "Convex optimization problems involving finite autocorrelation sequences," Mathematical Programming, Series A, vol. 93, no. 3, pp. 331-359, 2002.

[14] B. Dumitrescu, I. Tăbuş, and P. Stoica, "On the parameterization of positive real sequences and MA parameter estimation," IEEE Transactions on Signal Processing, vol. 49, no. 11, pp. 2630-2639, 2001. 
[15] B. Dumitrescu and C. Popeea, "Accurate computation of compaction filters with high regularity," IEEE Signal Processing Letters, vol. 9, no. 9, pp. 278-281, 2002.

[16] J. F. Sturm, "Using SeDuMi 1.02, a Matlab toolbox for optimization over symmetric cones," Optimization Methods and Software, vol. 11/12, no. 1-4, pp. 625-653, 1999, http://sedumi. mcmaster.ca.

[17] S. Haykin, Adaptive Filter Theory, Prentice-Hall, Upper Saddle River, NJ, USA, 4th edition, 2002.

Bogdan Dumitrescu was born in Bucharest, Romania, in 1962. He received the M.S. and Ph.D. degrees in 1987 and 1993, respectively, from the "Politehnica" University of Bucharest, Romania. He is a Professor at the Department of Automatic Control and Computers, "Politehnica" University of Bucharest. He held visiting research positions at the Institut National Polytechnique de Grenoble, France $(1992,1994,1996)$, and

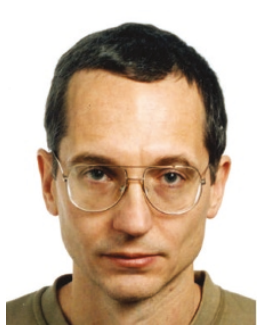
Tampere International Center for Signal Processing, Tampere University of Technology, Finland (1999-2001, 2003-2005). His scientific interests are in numerical methods, optimization, and their applications to signal processing. He is a Member of the IEEE.

Robert Bregović was born in Varaždin, Croatia, in 1970. He received the Diploma Engineer and the M.S. degrees in electrical engineering from the Faculty of Electrical Engineering and Computing, University of Zagreb, Croatia, in 1994 and 1998, respectively, and the Doctor of Technology degree (with honors) in information technology from the Tampere University of Technology in 2003. From 1994 to 1998, he was

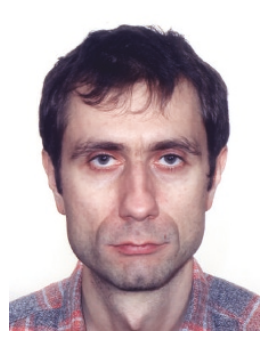
an Assistant at the Department of Electronic Systems and Information Processing, Faculty of Electrical Engineering and Computing, University of Zagreb. In 1999, he was a Visiting Researcher at the Tampere International Center for Signal Processing, Institute of Signal Processing Laboratory, Department of Information Technology, Tampere University of Technology. From January 2000 to August 2003, he was a Researcher, and since September 2003, he has been a post-doctoral Researcher at the same laboratory. His research interests are in digital signal processing, especially in multirate signal processing and digital filterbanks, and in generating optimization procedures for designing digital filters and filterbanks for various applications, as well as optimizing DSP algorithms for digital signal processor implementations.

Tapio Saramäki has received the Diploma Engineer and Doctor of Technology degrees (both with honors) in electrical engineering from the Tampere University of Technology (TUT), Finland, in 1978 and 1981, respectively. He is currently a Professor of Signal Processing and a Docent of Telecommunications in TUT. Furthermore, he is also a cofounder and a system-level designer of VLSI Solution Oy and the President of

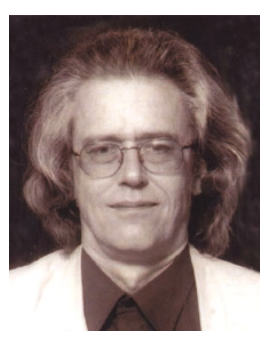
Aragit Oy Ltd., both in Tampere, Finland. He has been a Visiting Professor five times with the University of California, Santa Barbara, once with the California Institute of Technology, Pasadena, and once with the National University of Singapore. His research interests are in digital signal processing, especially filter and filter bank design, VLSI implementations, and communications applications, as well as approximation and optimization theories. He has written more than 250 international journal and conference articles and various international book chapters, and he holds three worldwide used patents. He has received the 1987 IEEE Guillemin-Cauer Award and two other best paper awards. He is a Fellow of the IEEE and the Russian A. S. Popov Society. He was an Associate Editor of some IEEE Transactions and is currently an Associate Editor of Circuits, Systems, and Signal Processing. He was also a Distinguished Lecturer of the IEEE Circuits and Systems Society and the Chairman of the IEEE Circuits and Systems DSP Technical Committee. 